\title{
Lexical Entrainment of Real Users in the Let's Go Spoken Dialog System
}

\author{
Gabriel Parent, Maxine Eskenazi \\ Language Technologies Institute, Carnegie Mellon University, Pittsburgh PA USA \\ gparent@cs.cmu.edu, max@cs.cmu.edu
}

\begin{abstract}
This paper examines the lexical entrainment of real users in the Let's Go spoken dialog system. First it presents a study of the presence of entrainment in a year of human-transcribed dialogs, by using a linear regression model, and concludes that users adapt their vocabulary to the system's. This is followed by a study of the effect of changing the system vocabulary on the distribution of words used by the callers. The latter analysis provides strong evidence for the presence of lexical entrainment between users and spoken dialog systems.
\end{abstract}

Index Terms: spoken dialog systems, lexical entrainment

\section{Introduction}

The success of a conversation between a user and a spoken dialog system (SDS) is influenced by many of the SDS designer's choices. Some of those decisions concern the words that the system will use. Users may be confused by ambiguous terms in an SDS, while they also may not know precise but rare terms. Vocabularies constantly change in human dialog. Humans dynamically adapt their choice of words to one another to facilitate the flow of the conversation. This process is commonly referred to as lexical entrainment [1]. Wizard of $\mathrm{Oz}$ (WOZ) studies [2] have shown that users also tend to adapt to the "system" vocabulary. SDS designers have long known that the users who are successful at using an SDS are those who adapt to the system. Yet, to our knowledge, there is very little literature detailing manners and rates of the user adaptation process [3].

In this paper, we present the results from a study of lexical entrainment of real users of Let's Go, a bus information SDS [4]. More specifically, we answer two questions: do real users entrain to the SDS' vocabulary and how do users adapt when new system-spoken primes are introduced. These primes cover different concepts, and include nouns, verbs, adjectives and adverbs. Measures of entrainment (both presence and strength) are calculated for a total of 18981 transcribed dialogs $(197,801$ turns), spanning a period of over one year of use of the Let's Go SDS. We employ measures used in the literature, and introduce a new analysis of lexical entrainment based on word frequency before and after prime modification. The large quantity of human-transcribed data allows us to conclude that what others have observed in a WOZ set-up and in offline data studies holds, with strong statistical significance, for real SDS users; the callers do entrain to system primes. Finally, while one short dialog might not be enough to get a seasoned user to say the new system prime, observation over a period of three weeks shows positive evidence of longer-term adaptation.

\section{Background}

Human-human dialogue research has shown that in a conversation each speaker implicitly gravitates towards the other's manner of speaking [1] [2]. Levelt [5] proposed that the process was lexically driven, since a lexical item is chosen and then encoded at many levels so that both meaning and form substitutions are possible. Thus the adaptation is both an effort at aligning language and at aligning meaning, aiming at a productive conversation.

A novice SDS user might be more likely to adapt to the system than a frequent user, who should already have adapted. Levow [3] compared novice system users to "experts" to see how quickly the former adapted their speech. Her longitudinal analysis showed that novices can adapt word choice fairly quickly (also reducing their vocabulary). This lowered the out of vocabulary (OOV) rate, but did not raise their speech recognition scores. Again on the lexical level, in WOZ simulations, Gustafson and colleagues [6] detected lexical entrainment and determined that users adapted to a synonym of a word that they had already used if it was spoken by the WOZ, thus proving that a prime could be modified by the system. This can be useful if the user proposes a prime that is difficult for an automatic system to understand. The system could deal with the difficult word by guiding the user to a longer, more phonetically unique lexical entry. Another good reason to use entrainment strategies when designing an SDS is Nenkova and colleagues' [7] finding that successful entrainment of high frequency words in human-human dialogues has a high correlation with task success. They observed that where there is more entrainment, there are more overlaps and fewer interruptions, thus producing better dialogue flow and fewer chances for the system to make errors. Matessa [8] shows similar results, where the average message length in a dialog between a lexically "accommodating" agent (the agent picks the same words as the user) and the user is shorter than it is with a "non-accommodating" agent (the agent purposely picks different words from the user).

However, findings from a WOZ setup may not necessarily be replicated with the real users of an SDS. Ai et al [9] note that real users behave differently from paid users. Thus WOZ findings should be verified on an SDS with real users. Stoyanchev and Stent [10] [11] used a subset of Let's Go data (2184 dialogs) to study the level of entrainment of the user to syntactic primes used by the system. Results were obtained using raw automatic speech recognition (ASR) output. These studies included different lexical entries ( 2 verbs and 2 prepositions), and give early confirmation that entrainment may be present in real users' interaction with an SDS. While the present paper first confirms this observation, it goes actively beyond by directly manipulating the system primes. It also exploits a significant amount of data, thus affording observations of user adaptation over time.

\section{The Lets's Go vocabulary}

This study is based on dialogs between real users and the Let's Go bus information system [4]. The system is used nightly by Port Authority of Allegheny County bus riders. They call to obtain both bus schedule information and to find out what bus runs in a given neighborhood. Table 1 shows a sample dialog.

In order to study a variety of aspects of users' lexical entrainment, we first examined all of the existing system prompts and selected a set of key concepts that were likely to 


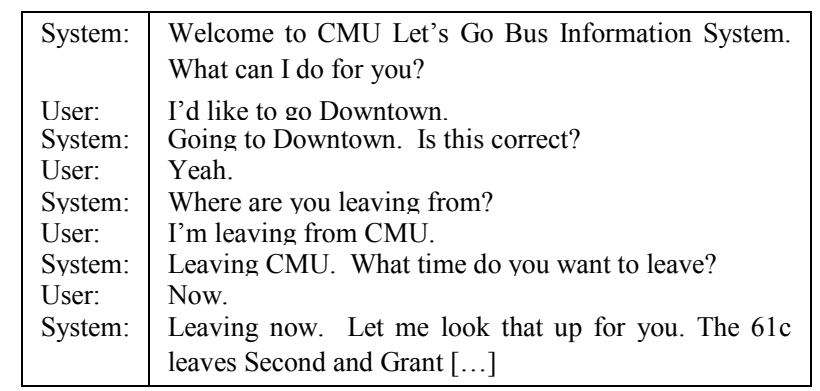

Table 1. Example of a dialog in Let's Go SDS

be expressed by the users and the systems in order for the dialog to be successful for example "bus immediacy" (e.g., "now", "the next bus") (Table 2). For each of these concepts, we selected a word in the existing vocabulary (V1) that was likely to be entrained by the users. There were 10 words in all. We built a second vocabulary (V2) which, for each of the 10 words we had selected, contained a corresponding word that was deemed to contain the same semantic content as the V1 word. For example, "immediately" was chosen as the word corresponding to "now". While V1 contains 10 words, V2 contains 8 ( 2 words in the second vocabulary replaced 4 words in the first vocabulary in an attempt to narrow V2). The words cover multiple classes of part-of-speech. It should be noted that we purposely selected right to be part of V2 even though it was also in V1 (see Section 5). We verified that all of the words were synthesized intelligibly, and recognized correctly. Since all of the dialogs were ultimately hand transcribed, the quality of the recognizer is not a factor of precision in our measure of lexical entrainment.

\section{Linear regression analysis of lexical entrainment}

\subsection{Methodology}

To obtain a baseline confirmation that real users of an SDS do indeed adapt to the system primes, we used the measure presented in [12]. The idea is to build a linear model of the prime/target distance and of the frequency of that pair. This model is built using the data in two orders: normal order, and random order (the user and system turns are shuffled

\begin{tabular}{|c|c|c|c|}
\hline Category of primes & Words in V1 & Words in V2 & POS \\
\hline \multirow{3}{*}{ Bus immediacy } & next & following & $\mathrm{ADJ}$ \\
\hline & previous & preceding & $\mathrm{ADJ}$ \\
\hline & now & immediately & ADV \\
\hline Action & leaving & departing & VB \\
\hline \multirow{2}{*}{$\begin{array}{l}\text { Domain } \\
\text { specific }\end{array}$} & route & \multirow{2}{*}{ itinerary } & \multirow{2}{*}{$\mathrm{N}$} \\
\hline & schedule & & \\
\hline \multirow{3}{*}{ Agreement } & okay & \multirow{3}{*}{ right } & \multirow{3}{*}{$\mathrm{ADJ}$} \\
\hline & correct & & \\
\hline & right & & \\
\hline \multirow{2}{*}{ System interaction } & help & assistance & $\mathrm{N}$ \\
\hline & query & request & $\mathrm{N}$ \\
\hline
\end{tabular}

Table 2. Vocabulary used by Let's Go for this experiment

randomly). If the models for the two orders are the same, this means that the order does not affect the relationship between the two variables. However, our hypothesis is that a user will reuse a word more often immediately after the system has said it, and thus the model with the normal order should have a different slope than the random model. This idea is also applied in [13] as a way of finding evidence of entrainment on syntactic features.

We examined one year of Let's Go real caller data $(18,081$ dialogs, referred to as D1 hereafter) containing the V1 primes. These dialogs were transcribed by a crowd of workers through the Amazon Mechanical Turk (AMT) platform [14].

\subsection{Results}

To obtain the data for our linear model, we gather data points by obtaining, for every V1 prime that the system used, the uptake of that prime by the user within a window of $\mathrm{N}$ turns. Table 3 presents an example of two data points obtained in this manner way. The first one $([1,1])$ is obtained because the user said the word itinerary one turn after the system did and the second one $([2,1])$ is obtained because the word help is used by the user two turns after the system used it. If the user entrained to the system vocabulary, there should be more data points with a small distance, which the linear model should be able to capture.

\begin{tabular}{|l|l|l|}
\hline \multicolumn{1}{|c|}{ Speaker } & \multicolumn{1}{|c|}{ Transcripts } & $\begin{array}{c}\text { Data } \\
\text { points }\end{array}$ \\
\hline System: & $\begin{array}{l}\text { I am an automated agent that can give you } \\
\text { itinerary information about buses. You can ask } \\
\text { for help at any time. What can I do for you? }\end{array}$ & \\
\hline User: & I'd like the itinerary for the 61C. & {$[\mathbf{1 , 1}]$} \\
\hline System: & Where are you leaving from? & \\
\hline User: & Help & {$[\mathbf{2 , 1 ]}$} \\
\hline
\end{tabular}

Table 3. Example of data points for the linear regression

After determining these data points for all of the dialogs, we can build a linear regression model and obtain slopes that express a linear relationship between the two variables. Pvalues that express the probability that this relationship would exist by chance, as well as an $\mathrm{R}$ which represents how much the two variables correlate were also calculated. Table 4 shows the results for the normal order and the random order baselines.

\begin{tabular}{|l|c|c|c|c|c|c|}
\hline \multirow{2}{*}{ Data } & \multicolumn{2}{|c|}{ N=5 } & \multicolumn{2}{c|}{ N=10 } & \multicolumn{2}{c|}{ N=15 } \\
\cline { 2 - 7 } & Slope & $\mathbf{R}$ & Slope & $\mathbf{R}$ & Slope & $\mathbf{R}$ \\
\hline $\begin{array}{l}\text { Normal } \\
\text { order }\end{array}$ & $\mathbf{- 0 . 7 3}$ & $\mathbf{- 0 . 1 8}$ & $\mathbf{- 0 . 3 2}$ & $\mathbf{- 0 . 1 8}$ & $\mathbf{- 0 . 1 7}$ & $\mathbf{- 0 . 1 5}$ \\
\hline $\begin{array}{l}\text { Random } \\
\text { order } \\
\text { (Baseline) }\end{array}$ & $\mathbf{- 0 . 0 8}$ & $\mathbf{- 0 . 0 2}$ & -0.01 & -0.01 & 0.01 & 0.01 \\
\hline
\end{tabular}

Table 4. Slopes and Rs of the linear regression for different window size. Bold values are statistically significant $(\boldsymbol{p}<\mathbf{0 . 0 0 1})$

\subsection{Discussion}

The baseline (randomized turn order) confirms that our measure is valid, since the gradients of the linear regression for each of the 3 different window sizes are all close to 0 and all the Rs are small (no linear relationship exists between the two variables). On the other hand, in the normal order, the two variables (prime/target distance and frequency of response) appear to be weakly correlated (all $|\mathrm{R}| \mathrm{s}$ are above 0.15 ). The slopes indicate that the frequency of prime/target pairs sepa- 
rated by a small distance is higher that of pairs at a larger distance.

These results are comparable to [12]; the distance between a prime/target pair has an effect on the frequency of such a pair. However, this approach raises several questions. One is that our measure does not take into account the fact that some words have more synonyms (and thus more chance of fortuitous system/user alignment). Also, as Ward and Litman [12] mention, the size of an optimal window to measure entrainment, and the underlying reason for that window size are still open questions.

More generally, a major issue is that this analysis does not take into account the prior distribution of the words. Section 5 presents another kind of analysis that examines two word distributions: before and after a change in primes. If the system's vocabulary has an influence on the user's choice of words, the word distributions should be different.

\section{How do users react to a change in primes?}

\subsection{Methodology}

Without informing our callers, we changed the Let's Go vocabulary from V1 to V2. We took out all $10 \mathrm{~V} 1$ words from all system prompts (except for right, as explained in 5.3), so that the results would only reflect the effect of the intended V2 primes. We ran the new set of prompts for 3 weeks $(900$ dialogs, referred as $D 2$ hereafter), which were all transcribed by an expert transcriber. The analysis is based on the frequency of words in the two vocabularies. If the user does not entrain to the system vocabulary, the distribution of V1 words in the user's speech should be the same before and after the change. On the other hand, if there is lexical entrainment, we should see more V2 words after the change, since they had been spoken by the system.

\subsection{Results}

Table 5 shows the frequencies of words in both vocabularies in D1 (one year using V1 primes) and D2 (3 weeks using V2 primes). The results for the dataset D2 are also shown in Figure 1 to allow an easier comparison: the proportion occupied by each word on one line corresponds to its relative frequency in D2. In order to give an order of magnitude of how many times each word was used in D2, we also included the count of the V1 words next to their corresponding label. In order to have an idea of the evolution of user entrainment over time, we calculated for each day the proportion of words used that were from V2 compared to V1. For example, a proportion of 0.4 indicates that out of 10 words used by the caller, 4 were from V2.

\subsection{Discussion}

From Table 5, we can see that the words we selected to be part of V2 almost never occurred in D1. One explanation is that the seasoned callers are well-aligned with the SDS, which is also corroborated in Section 4 (We should note that we do not have caller-caller identification and thus cannot verify who is a repeat caller). The homogeneity of the words used in D1 (almost entirely V1 words) gives further strength to our results. The shift of word frequency toward words in V2, shown in Figure 1, can be tested using a paired t-test. We tested if the frequency shifts from D1 to D2 for words in V1 and V2 were from the same distribution. We obtained a p-value of 0.0011 , thus allowing us to reject the null-hypothesis that words in V1 and V2 behave similarly after the prompts change. The Table 5 also makes the difference clear. For example, the word preceding was used 0 times over a period of 1 year prior to our changes and 96 times in the 3 weeks that followed the insertion of that word in the various prompts of the system.

\begin{tabular}{|l|c|r|}
\hline \multicolumn{1}{|c|}{ Words } & D1 Freq. (\% rel. Freq) & D2 freq (\% rel. Freq) \\
\hline V1: next & $13204(99.9 \%)$ & $492(82.9 \%)$ \\
V2: following & $3(0.1 \%)$ & $101(17.1 \%)$ \\
\hline V1: previous & $3066(100 \%)$ & $78(44.8 \%)$ \\
V2: preceding & $0(0 \%)$ & $96(55.2 \%)$ \\
\hline V1: now & $6241(99.8 \%)$ & $237(80.1 \%)$ \\
V2: immediately & $10(0.2 \%)$ & $59(19.9 \%)$ \\
\hline V1:leaving & $4843(98.4 \%)$ & $165(70.8 \%)$ \\
V2: departing & $81(1.6 \%)$ & $68(29.2 \%)$ \\
\hline V1: route/schedule & $2189(99.9 \%)$ & $174(94.5 \%)$ \\
V2: itinerary & $2(0.1 \%)$ & $10(5.5 \%)$ \\
\hline V1: okay/correct & $1371(49.3 \%)$ & $48(27.7 \%)$ \\
V2: right & $1409(50.7 \%)$ & $125(72.3 \%)$ \\
\hline V1: help & $2189(99.9 \%)$ & $17(65.3 \%)$ \\
V2: assistance & $1(0.1 \%)$ & $9(34.7 \%)$ \\
\hline V1: query & $6256(99.9 \%)$ & $70(20.4 \%)$ \\
V2: request & $3(0.1 \%)$ & $272(79.6 \%)$ \\
\hline
\end{tabular}

Table 5. V1 and V2 word frequencies in D1 and

However, it seems that not every word was entrained to the same extent. The prime, itinerary, was the one that callers used the least (from a relative frequency of $0.09 \%$ in D1 to $5.75 \%$ in D2). This can be explained by the fact that this word is generally less frequent, domain-specific and also ambiguous in the context of a bus information system (it can represent the time when a bus runs, as well as a bus route).

The word request is particularly interesting. Its frequency goes from $0.05 \%$ to $79.53 \%$. One possible explanation is that its counterpart in V1, query, is less frequent, thus less known to callers and so probably less natural for them It should be noted that this prime provides interaction with the system (it's

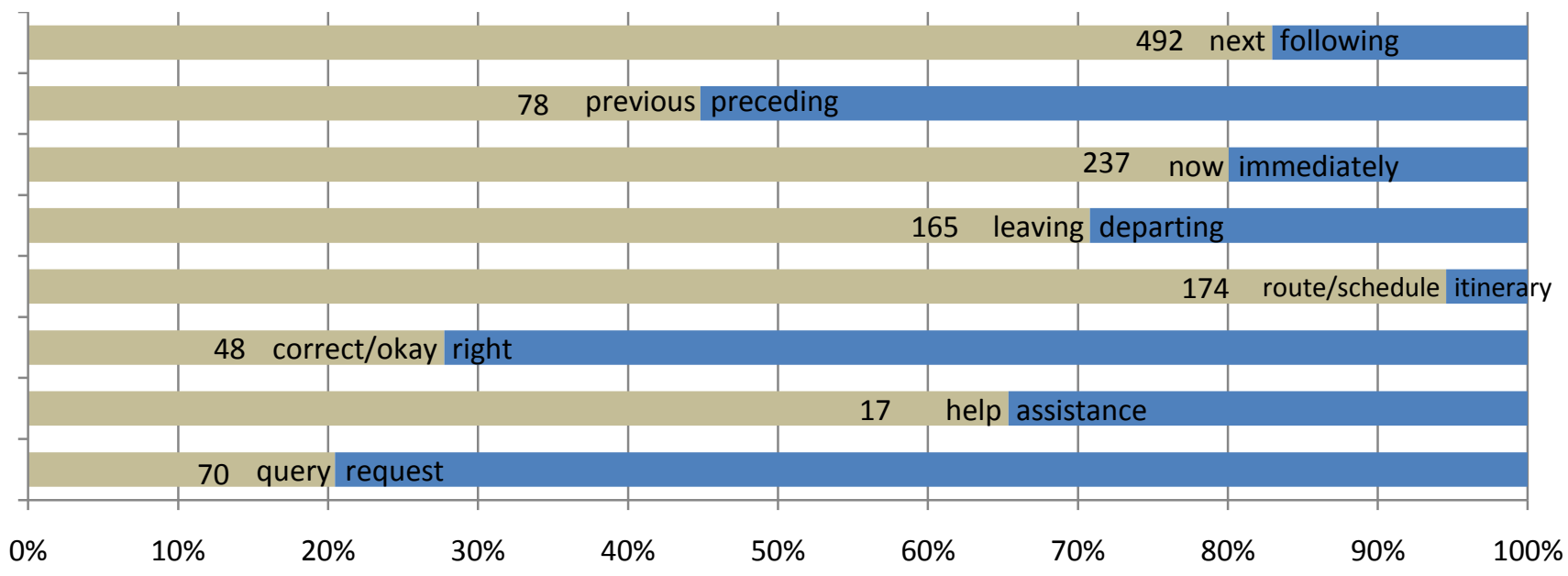




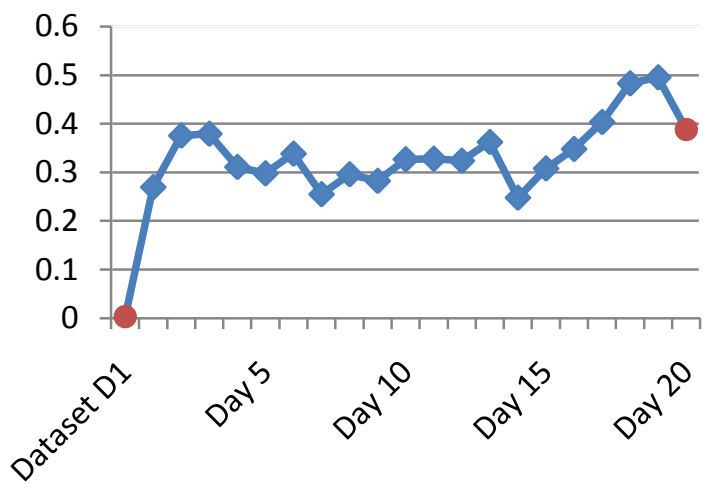

Figure 2. Proportion of words of $V 2$ in the target words

mostly used in "start a new request" to restart the dialog). It may be the case that the users entrain more to this type of prime, since it affects the intrinsic behavior of the system, thinking that the system is probably the best reference on how to name a concept like "start a new request".

The most entrained word (request) and the least entrained one (itinerary) are both nouns. Also, there does not seem to be a pattern in the strength of entrainment for the different parts-of-speech (POS), and thus we do not have enough evidence to conclude that POS could be used to predict entrainment. However, we do note that it seems that more frequent words are more easily entrained. As an example, itinerary, which is much less frequent in day-to-day speech in this domain than schedule, is not chosen by many users. The opposite happens for request and query, query being the infrequent term in this case.

The evolution of lexical entrainment between users and the SDS is shown on Figure 2. Although there isn't a clear upward pattern, there seems to be a positive tendency, especially in the third week. There are also 3 points where the curve goes down, which correspond to the 3 weekend periods during the study. This is likely to be an indication that some of our users only call on the weekend, and thus take more time to adapt to the system vocabulary, although again we cannot confirm this without caller-identification information.

\section{Conclusion}

This paper presents an experiment aimed at characterizing the lexical entrainment of real users calling the Let's Go Spoken Dialog System. We first used a convergence measure to determine lexical entrainment of the system users. The results indicate that users tend to adapt to primes that are in system prompts, and are more likely to do so in the first few turns following that system prime. Another approach that is based on the word frequency of words in two different vocabularies was presented. Some V2 words that had a very low frequency in D1 have a higher frequency than their V1 counterpart. This adaptation seems to be unequal across different words; in our case, the users seem to entrain less to unnatural and harder words. Also, although an abrupt change can be observed in word frequencies immediately after the transition from V1 to $\mathrm{V} 2$, the proportion of $\mathrm{V} 2$ words continues to increase after the second week of the study. This may indicate that the user needs more exposure than just one call to fully entrain to the system's primes.

Future work will examine the contexts in which the primes were used. For example, help and assistance were meant to be used in the same unique context of the user needing help. More complex manipulation could determine if entrainment also holds for prime/target pairs that are not meant to be used in the same context. An example would be if the system, ad- dressed the user with a prompt such as "In the next minutes, I will be asking you some questions" where the word next is used by the system to refer to a time period. By excluding the primes next and following from the normal bus information prompts ("Do you want the next bus?"), only the first greeting would contain the prime. However, such an approach would need some type of compensation for the prior distribution of the two words. This experiment would also be harder to implement in a natural way in a bus information system since the domain is limited. We are beginning to develop an SDS with a wider domain of application that will afford this type of study. That platform will thus allow us to make finer adjustments, and investigate more complex aspects of lexical entrainment. It will also provide "caller id", which will allow us to individualize the analysis, and eventually the manipulations.

Finally, an effective SDS should not only have the user entrain to its primes, but also detect user primes and modify its own prompts to use them. This is the next step in our effort to endow SDS with entrainment capabilities.

\section{Acknowledgements}

This work was funded by NSF grant IIS-0914927 "LexE: Using two-part lexical entrainment for more efficient and reliable spoken dialogue systems". The opinions expressed in this paper do not necessarily reflect those of NSF. We would like to thank Adam Skory and Susi Burger for their help in transcription expertise.

\section{References}

[1] Garrod, S. and A. Anderson, 1987, Saying what you mean in dialogue: A study in conceptual and semantic co-ordination, Cognition, *27*, p.181-218

[2] Brennan, S., 1996, Lexical entrainment in spontaneous dialog, Proceedings of ISSD, p. 41-44.

[3] Levow, G-A, 2003, Learning to Speak to a Spoken Language System: Vocabulary Convergence in Novice Users, in Proceedings of 4th SIGdial Workshop on Discourse and Dialogue, 9153 .

[4] Raux, A., Langner, B. Bohus, D. Black, A W, Eskenazi,M., 2005, Let's Go Public! Taking a Spoken Dialog System to the Real World, Interspeech 2005 Lisbon, Portugal.

[5] Levelt, WJM, 1996, A theory of lexical access in speech production, Proc $16^{\text {th }}$ Conference on Computational Linguistics, vol 1.

[6] Gustafson, J., Larsson, A., Carlson, R., Hellman, K., 1997, How do System Questions Influence Lexical Choices in User Answers?, Proceedings of EUROSPEECH'97.

[7] Nenkova, A., Gravano, A., Hirschberg, J., 2008, High frequency word entrainment in spoken dialogue, Proceedings of ACL/HLT 2008 (short paper), pages 169-172.

[8 Matessa, M, 2003, Measure of Adaptive Communication, Second Workshop on Empirical Evaluation of Adaptive Systems

[9] Ai, H., Raux, A., Bohus, D., Eskenazi, M., and Litman, D. (2007) Comparing Spoken Dialog Corpora Collected with Recruited Subjects versus Real Users, 8th SIGDial Workshop on Discourse and Dialogue, Antwerp, Belgium.

[10] Stoyanchev, S., Stent, A., 2009, Concept Form Adaptation in Human-Computer Dialog. Proc. SIGDIAL conference, London.

[11] Stoyanchev, S., Stent, A., 2009, Lexical and Syntactic Priming and Their Impact in Deployed Spoken Dialog Systems, Proc. NAACL09

[12] Ward, A., Litman, D., 2007 "Automatically measuring lexical and acoustic/prosodic convergence in tutorial dialog corpora", Proceedings ISCA SLaTE Workshop, p. 57-60

[13] Reitter, D. , Keller, F. and Moore, J., 2006. "Computational Modelling of Structural Priming in Dialogue", Proceedings of the Human Language Technology Conference of the NAACL, p. $121-124$

[14] Crowdsourcing for Natural Language Technologies, LTI, CMU. http://crowdsourcing.lti.cs.cmu.edu 